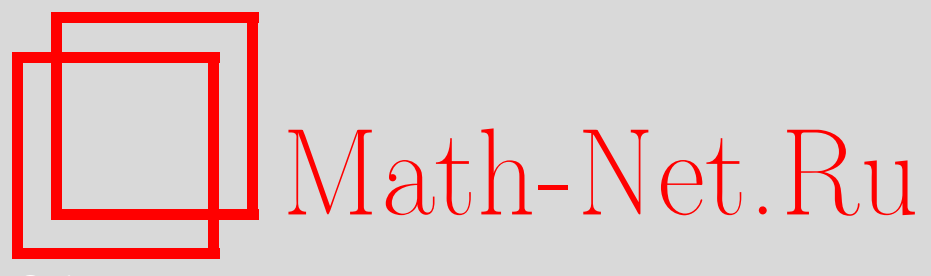

Я. В. Новак, О наилучшем локальном приближении наипростейшими дробями, Матем. заметки, 2008, том 84, выпуск 6, 882-887

DOI: https://doi.org/10.4213/mzm4168

Использование Общероссийского математического портала Math-Net.Ru подразумевает, что вы прочитали и согласны с пользовательским соглашением http: //www . mathnet.ru/rus/agreement

Параметры загрузки:

IP : 54.196 .121 .252

26 апреля 2023 г., 17:16:20

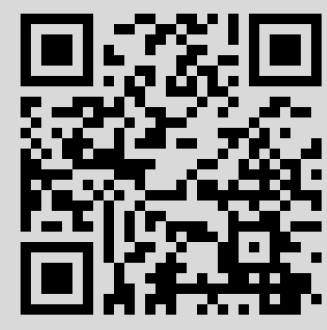


Том 84 выпуск 6 декабрь 2008

УДК 517.538 .5

\section{О наилучшем локальном приближении наипростейшими дробями}

\section{Я. В. Новак}

В работе приводятся две теоремы о локальном наилучшем приближении наипростейшими дробями, т.е. логарифмическими производными алгебраических многочленов с комплексными коэффициентами. В теореме 1 получен аналог известной теоремы Бернштейна об описании функций $n$ раз непрерывно дифференцируемых на отрезке $\Delta \subset \mathbb{R}$ в терминах локальных приближений в равномерной метрике алгебраическими многочленами. Теорема 2 дает описание наипростейшей дроби Паде как предела последовательности наипростейших дробей наилучшего равномерного приближения и является аналогом известного результата Уолша о классических дробях Паде.

Библиография: 6 названий.

1. Введение. Наипростейшей дробъю степени $n$ (термин предложен Е. П. Долженко) называется рациональная функция вида

$$
r_{n}(z)=\sum_{k=1}^{n} \frac{1}{z-a_{k}}=\frac{P^{\prime}(z)}{P(z)}, \quad\left\{a_{k}\right\}_{k=1}^{n} \subset \mathbb{C}, \quad n \in \mathbb{N}:=\{1,2, \ldots\},
$$

где $P(z):=\left(z-a_{1}\right) \cdots\left(z-a_{n}\right)$.

Как аппарат приближения наипростейшие дроби активно исследуются с 1999 года, когда В.И. и Д. Я. Данченко (см. [1]) установили возможность равномерного приближения с произвольной точностью наипростейшими дробями каждой (комплексной) функции, непрерывной на компакте и аналитической в его внутренних точках, при условии, что этот компакт не разбивает плоскость (аналог известной теоремы С.Н. Мергеляна). Некоторые другие задачи теории равномерного приближения наипростейшими дробями изучались в работах [2]-[4]. Например, в [2] установлен аналог известной теоремы Джексона о приближении непрерывных на отрезке функций с заданным модулем непрерывности, а в [3] доказана возможность приближения наипростейшими дробями функций, непрерывных на вещественной оси $\mathbb{R}$, которые исчезают на бесконечности.

В настоящей работе приводятся две теоремы о локальных приближениях наипростейшими дробями. Для их формулировки введем следующие обозначения.

Как обычно, $C(I)$ - линейное пространство непрерывных на отрезке $I=[\alpha, \beta] \subset \mathbb{R}$, $\alpha<\beta$, функций $f$ с нормой $\|f\|_{I}=\max _{t \in I}|f(t)|, C^{n}(I)$ - множество $n$ раз непрерывно дифференцируемых функций на отрезке $I$. Через $\mathbf{S R}_{n}$ мы обозначаем множество 
всех наипростейших дробей степени не выше $n$; при $n=0$ по определению оно состоит из единственной функции тождественно равной нулю.

Через

$$
\rho_{n}(f ; I):=\inf \left\{\left|f-r_{n}\right|_{I}: r_{n} \in \mathbf{S R}_{n}\right\}
$$

обозначим величину наилучшего равномерного приближения функции $f \in C(I)$ наипростейшими дробями степени не выше $n$. Если в определении величины $\rho_{n}(f ; I)$ заменить множество $\mathbf{S R}_{n}$ на множество $\mathbf{P}_{n}$ всех алгебраических многочленов степени не выше $n$, то получим определение величины $E_{n}(f ; I)$. Для функции $f$, интегрируемой на отрезке $I$ и для фиксированной точки $x \in I$, положим $\alpha(f, x ; t):=\int_{x}^{t} f(\tau) d \tau$. Функция $\alpha(f, x ; t)$, очевидно, принадлежит пространству $C(I)$.

Пусть $a, b \in \mathbb{R}, a<b, \Delta:=[a, b]$, и пусть $n \in \mathbb{N}$. В принятых обозначениях имеет место следующий результат.

ТЕОрема 1. Для того чтобы непрерывная функиия $f(t)$ на отрезке $\Delta$ была на нем $n$ раз непрерывно дифберенцируемой, необходимо и достаточно, чтобъ существовала функиия $\lambda \in C(\Delta)$ такая, что

$$
\frac{\rho_{n}(f ;[\alpha, \beta])}{(\beta-\alpha)^{n}} \rightarrow \lambda(x), \quad a \leqslant \alpha<x<\beta \leqslant b,
$$

равномерно по $x$ при $\alpha, \beta \rightarrow x$. Если последнее условие выполнено, то имеет место тождество

$$
n ! 2^{2 n-1} \lambda(x) \equiv\left|\frac{d^{n}}{d t^{n}}\left(f(t) e^{\alpha(f, x ; t)}\right)_{t=x}\right| .
$$

Следует заметить, что выражение под модулем в (1) (для краткости, обозначим его через $\left.f^{[n]}(x)\right)$ после упрощений принимает вид, не содержащий функции $e^{\alpha(f, x ; t)}$. Это делается с помощью рекуррентных соотношений

$$
f^{[n+1]}(x)=\frac{d}{d x} f^{[n]}(x)+f(x) \cdot f^{[n]}(x), \quad f^{[0]}(x):=f(x) .
$$

Теорема 1 является аналогом известного результата Бернштейна [5] о том, что для принадлежности непрерывной функции $f(t)$ классу $C^{n}(\Delta)$ необходимо и достаточно, чтобы выполнялось следующее условие:

$$
E_{n-1}(f ;[\alpha, \beta])(\beta-\alpha)^{-n} \rightarrow \lambda(x), \quad a \leqslant \alpha<x<\beta \leqslant b,
$$

равномерно по $x$ при $\alpha, \beta \rightarrow x$, где $\lambda \in C(\Delta)$; если это условие выполнено, то имеет место равенство

$$
n ! 2^{2 n-1} \lambda(x) \equiv\left|f^{(n)}(x)\right| .
$$

Для формулировки второй теоремы напомним определение наипростейшей дроби Паде.

При $n \geqslant 1$ наипростейшей дробъю Паде порядка $n$ функции $f(z), z \in \mathbb{C}$, в точке $b$, в некоторой окрестности $U$, в которой $f(z)$ однозначна и аналитична, называется наипростейшая дробь $R_{n}(f, b ; \cdot) \in \mathbf{S R}_{n}$ со свойством:

$$
f(z)-R_{n}(f, b ; z)=O\left((z-b)^{n}\right) \quad \text { при } \quad z \rightarrow b .
$$


Известно, что дробь $R_{n}(f, b ; z)$ существует, единственна и совпадает с логарифмической производной $n$-й частичной суммы ряда Тейлора функции $e^{\alpha(f, b ; z)}$. Здесь $\alpha(f, b ; z)=\int_{b}^{z} f(\tau) d \tau$, где интегрирование ведется по любому спрямляемому пути в некоторой односвязной окрестности точки $b$, ведущему из $b$ в $z$. Этот факт установлен в работе [2], а существование и единственность - независимо в [1].

Пусть функция $f(z)$ однозначна и аналитична в некоторой окрестности $U$ точки $b$, $R_{n}(\varepsilon, z)$ - наипростейшая дробь ее наилучшего равномерного приближения в круге

$$
K(\varepsilon)=\{z \in \mathbb{C}:|z-b| \leqslant \varepsilon\} \subset U
$$

(если она не единственна, то фиксируем любую из них), $R_{n}(z):=R_{n}(f, b ; z)$ - наипростейшая дробь Паде порядка $n$ функции $f(z)$ в точке $b$. В принятых выше обозначениях имеет место следующий результат.

ТЕОРЕма 2. Функиии $R_{n}(\varepsilon, z)$ сходятся $\kappa$ функиии $R_{n}(z)$ при $\varepsilon \rightarrow 0+$ равномерно на компактных подмножествах комплексной плоскости $\mathbb{C}$, не содержащих полюсов функции $R_{n}(z)$.

Эта теорема является аналогом известного результата Уолша [6] о том, что аппроксимации Паде являются локально наилучшими рациональными аппроксимациями.

2. Вспомогательные результаты. Ниже

$$
\begin{gathered}
C_{1}(r)=\left((1+r) e^{r}\right)^{-1}, \quad C_{2}(r)=\left(1+2 r e^{r}\right) e^{r}, \quad r \in \mathbb{R}, \quad r \geqslant 0, \\
d:=\max \{\beta-x, x-\alpha\} .
\end{gathered}
$$

Следующая лемма и ее следствие для случая $\eta=1 / 2$ доказаны в [2] (см. также [4]). Для общего случая доказательство проводится по той же схеме.

Лемма 1. Пусть $f, g \in C(I), G(t)=1+\alpha(g, x ; t), G(t) \neq 0$ на $I$,

$$
\delta:=\left\|g-f e^{\alpha(f, x ; \cdot)}\right\|_{I}, \quad \varepsilon:=\left\|\frac{g}{G}-f\right\|_{I}, \quad \eta:=\delta d \cdot \exp \left(d\|f\|_{I}\right)<1 .
$$

Тогда

$$
\varepsilon \leqslant\left((1-\eta) C_{1}\left(d\|f\|_{I}\right)\right)^{-1} \cdot \delta, \quad \delta \leqslant C_{2}\left(d\|f\|_{I}\right) \cdot \varepsilon
$$

nрu $\varepsilon \leqslant\|f\|_{I}$.

СлеДСтвиЕ. Пусть $f \in C(I) \backslash \mathbf{S R}_{n+1}, n=0,1, \ldots$,

$$
\eta:=E_{n}\left(f e^{\alpha(f, x ; \cdot)}\right) d \cdot \exp \left(d\|f\|_{I}\right)<1 .
$$

Тогда

$$
(1-\eta) C_{1}\left(d\|f\|_{I}\right) \leqslant \frac{E_{n}\left(f e^{\alpha(f, x ; \cdot)} ; I\right)}{\rho_{n+1}(f ; I)} \leqslant C_{2}\left(d\|f\|_{I}\right) .
$$

Поскольку $C_{1}\left(d\|f\|_{I}\right) \rightarrow 1, C_{2}\left(d\|f\|_{I}\right) \rightarrow 1$, a $\eta=E_{n}\left(f e^{\alpha(f, x ; \cdot)}\right) d \cdot \exp \left(d\|f\|_{I}\right) \rightarrow 0$ при $d \rightarrow 0$, получаем следующий результат.

ЛЕмма 2. Пусть $f \in C(\Delta) \backslash \mathbf{S R}_{n+1}$, тогда при любом $x \in \Delta$

$$
\lim _{\alpha, \beta \rightarrow x} \frac{E_{n}\left(f e^{\alpha(f, x ; \cdot)} ; I\right)}{\rho_{n+1}(f ; I)}=1 .
$$


3. Доказательство теоремы 1. Сначала заметим, что функции $f(t)$ и $f e^{\alpha(f, x ; t)}$ строго одновременно принадлежат или не принадлежат классу $C^{n}(\Delta)($ см. [4; с. 32]).

Пусть $f \in C^{n}(\Delta)$, тогда также $f e^{\alpha(f, x ; t)} \in C^{n}(\Delta)$. Применяя к последней функции теорему Бернштейна, получаем, что

$$
E_{n-1}\left(f e^{\alpha(f, x ; t)} ;[\alpha, \beta]\right)(\beta-\alpha)^{-n} \rightarrow \lambda(x), \quad a \leqslant \alpha<x<\beta \leqslant b,
$$

равномерно по $x$ при $\alpha, \beta \rightarrow x$, где непрерывная функция $\lambda(x)$ определяется равенством (1). Сравнивая с леммой 2, получаем необходимость условий теоремы 1.

Пусть теперь

$$
\rho_{n}(f ;[\alpha, \beta])(\beta-\alpha)^{-n} \rightarrow \lambda(x), \quad a \leqslant \alpha<x<\beta \leqslant b,
$$

равномерно по $x$ при $\alpha, \beta \rightarrow x$, где непрерывная функция $\lambda(x)$ определяется равенством (1). Снова воспользовавшись леммой 2, получаем справедливость условия теоремы Бернштейна для функции $f e^{\alpha(f, x ; t)}$, согласно которой последняя (а вместе с ней и сама функция $f(t))$ принадлежит классу $C^{n}(\Delta)$. Этим доказана достаточность условий теоремы 1.

4. Доказательство теоремы 2. Доказательство проводится по схеме подобной оригинальному доказательству Уолша [6]. Не теряя общности, будем считать, что $b=0$.

Пусть разложения в ряд Тейлора (при $|z|<\varepsilon$ ) функций $f(z)$ и $R_{n}(\varepsilon, z)$ имеют соответственно вид

$$
\begin{gathered}
f(z)=\sum_{k=0}^{\infty} a_{k} z^{k} \\
R_{n}(\varepsilon, z)=\frac{v_{1}+2 v_{2} z+\cdots+n v_{n} z^{n-1}}{1+v_{1} z+\cdots+v_{n} z^{n}}=\sum_{k=0}^{\infty} b_{k}(\varepsilon) z^{k}
\end{gathered}
$$

(мы нормировали знаменатель дроби $R_{n}(\varepsilon, z)$, придав ему значение 1 в точке $z=0$; в нуль, очевидно, он обращаться не может). Отсюда получаем равенство

$$
\left(1+v_{1} z+\cdots+v_{n} z^{n}\right) \sum_{k=0}^{\infty} b_{k}(\varepsilon) z^{k}=v_{1}+2 v_{2} z+\cdots+n v_{n} z^{n-1} .
$$

Приравнивая коэффициенты при соответствующих степенях $z^{j}, j=0, \ldots, n-1$, после упрощений, получим следующую систему $n$ уравнений (в которой $b_{k}(\varepsilon)=: b_{k}$ )

$$
\begin{aligned}
& v_{1}=b_{0}, \\
& -b_{0} v_{1}+2 v_{2}=b_{1}, \\
& -b_{n-2} v_{1}-b_{n-3} v_{2}-\cdots-b_{0} v_{n-1}+n v_{n}=b_{n-1} .
\end{aligned}
$$

Предположив $b_{k}(\varepsilon)$ известными, ее всегда можно решить, и при том однозначно, относительно неизвестных $v_{j}, j=1, \ldots, n$, поскольку ее определитель отличен от нуля (равен $n !)$. 
Пользуясь определением $R_{n}(z)$ мы можем записать

$$
R_{n}(z)=\frac{t_{1}+2 t_{2} z+\cdots+n t_{n} z^{n-1}}{1+t_{1} z+\cdots+t_{n} z^{n}}=\sum_{k=0}^{n-1} a_{k} z^{k}+\sum_{k=n}^{\infty} c_{k} z^{k} .
$$

Применяя ту же процедуру, что и выше, получим аналогичную предыдущей систему (такого же вида)

$$
\begin{aligned}
t_{1} & =a_{0}, \\
-a_{0} t_{1}+2 t_{2} & =a_{1}, \\
\cdots \ldots \ldots+\cdots \cdots \cdots \cdots & \ldots \cdots \\
-a_{n-2} t_{1}-a_{n-3} t_{2}-\cdots-a_{0} t_{n-1}+n t_{n} & =a_{n-1},
\end{aligned}
$$

которую точно также можно однозначно решить относительно неизвестных $t_{j}, j=$ $1, \ldots, n$, при известных коэффициентах $a_{k}$.

Используя экстремальное свойство $R_{n}(\varepsilon, z)$, имеем

$$
\max _{z \in K(\varepsilon)}\left|f(z)-R_{n}(\varepsilon, z)\right| \leqslant \max _{z \in K(\varepsilon)}\left|f(z)-R_{n}(z)\right| .
$$

Поскольку тейлоровские коэффициенты функций $f(z)$ и $R_{n}(z)$ при $k=0,1, \ldots, n-1$ совпадают, при $\varepsilon \rightarrow 0$

$$
\max _{z \in K(\varepsilon)}\left|f(z)-R_{n}(\varepsilon, z)\right|=O\left(\varepsilon^{n}\right) .
$$

Воспользовавшись интегральным представлением, получим

$$
a_{k}-b_{k}(\varepsilon)=\frac{1}{2 \pi i} \int_{|z|=\varepsilon} \frac{f(z)-R_{n}(\varepsilon, z)}{z^{k+1}} d z, \quad a_{k}-b_{k}(\varepsilon)=O\left(\varepsilon^{n-k}\right),
$$

и, таким образом, $a_{k}-b_{k}(\varepsilon) \rightarrow 0, \varepsilon \rightarrow 0$, при $k=0,1, \ldots, n-1$.

Последнее обстоятельство позволяет нам утверждать, что решения $t_{j}=t_{j}\left(a_{0}, \ldots\right.$, $\left.a_{n-1}\right)$ равномерно стремятся к решениям $v_{j}=v_{j}\left(b_{0}, \ldots, b_{n-1}\right), j=1, \ldots, n$, при $\varepsilon \rightarrow 0+$ (для этого достаточно воспользоваться известными формулами Крамера для решений обеих линейных систем), и как следствие мы получаем заключение теоремы 2 .

Пользуясь случаем, автор выражает благодарность А. В. Покровскому за постановку задач и помощь в работе над статьей, а также О. Н. Косухину за ряд полезных замечаний по поводу ее первоначального варианта.

\section{СПИСОК ЦИТИРОВАННОЙ ЛИТЕРАТУРЫ}

[1] В. И. Данченко, Д. Я. Данченко, “О приближении наипростейшими дробями”, Матем. заметки, 70:4 (2001), 553-559.

[2] О.Н. Косухин, "Об аппроксимационных свойствах наипростейших дробей”, Вестн. Моск. ун-та. Сер. 1. Матем., мех., 2001, № 4, 54-59.

[3] П.А. Бородин, О.Н. Косухин, "О приближении наипростейшими дробями на действительной оси”, Вестн. Моск. ун-та. Сер. 1. Матем., мех., 2005, № 1, 3-8. 
[4] О.Н. Косухин, О некоторых нетрадиционных методах приближения, связанных с комплексными полиномами, Дис. ... канд. физ.-матем. наук, МГУ, М., 2005.

[5] С. Н. Бернштейн, "К вопросу о локальном наилучшем приближении функций", Докл. AH CCCP, 26:9 (1940), 839-842.

[6] J. L. Walsh, "Padé approximants as limits of rational functions of best approximation", J. Math. Mech., 13:2 (1964), 305-312.

Я. В. Новак

Поступило

Институт математики НАН Украины

23.10.2007

E-mail: novak@imath.kiev.ua 